\title{
Microsurgery Practice in Developing Countries
}

\author{
Tuan Van Huynh, MD* \\ Department of Burn and Reconstructive Surgery, Trauma and Orthopaedics Hospital 175, Ho Chi Minh City, Vietnam
}

$\longrightarrow$ lobally, microsurgery has advanced great strides on the path of development. Microsurgery has become one of the routine approaches of treatment in many resource-abundant surgical centers, where a countless number of patients are daily treated for their injuries or defects by this technique.

The progress of microsurgery in developing low-income countries is very much slower compared to that in the developed countries. The implementation and development of microsurgery in resource-limited settings are really tough because of so many obstacles. The initial investment, which involves purchase of equipment, such as surgical microscopes, specialized micro-instrument sets, etc., is quite expensive. In addition, consumable supplies, such as microsurgical sutures, micro-hemoclip, as well as post-operative monitoring devices, are also very costly, and some of them are not even available in resource-limited settings.

Training and development of skills of microsurgical surgeons are also significant issues. Microsurgery is a technical skill that requires specialized training and practice. Hence, in order to perform micro-surgical techniques with high efficiency, the young microsurgical surgeons, subsequent to their basic training courses, must participate in many advanced courses. It is most important that they must practice their skills regularly. The specific skills in microsurgery include both microsurgical anastomosis as well as microsurgical dissection. Not to mention that successful outcomes require something more than just the skills used under the microscope. In order to maintain and improve these skills, the same should, preferably, be practiced on real patients. However, there is a paradox that, according to minimum standards of medical ethics, it is difficult to be accepted when we follow a certain therapy for our patients, while we cannot guarantee that the outcomes from this therapy are the best, with our capacity. Experimental micro-surgical models may be considered as the alternative. Here again, there are many complications. The living animal models have great advantages, such as closer to reality and allowing to practice the skills of both microsurgical anastomosis and microsurgical dissection. However, they are expensive, bulky and inconvenient; and hence it is really difficult to continue frequently in resourcelimited settings. On the other hand, the non-living models are cheap, flexible and easier to apply, but the efficiency of skill improvement is not high and practically not allowing to practice the skill of microsurgical dissection. Training and organizing of microsurgical teams are also difficult. Since microsurgery is a collective work, it is not possible to develop microsurgery regularly by relying on some individually trained microsurgeons. In fact, it is not easy to train microsurgical teams synchronously in developing countries.

The necessities for application of microsurgical techniques for treatment of patients are real and not limited by social and economic conditions. Today, microsurgery has become an important part of the modern surgeon's arsenal, particularly for plastic and reconstructive surgeons. Even though microsurgery may not be the best solution for all reconstructive dilemmas, it can still offer them an important tool for treating large tissue defects and complex wounds. In some situations, microsurgery is found to be an irreplaceable solution.

In developing low-income countries, in order to become capable to overcome obstacles and successfully develop microsurgery in daily professional activities in resource-limited settings, the health managers and surgeons, who are dedicated to their works, in addition to determination, also need to concentrate on suitable methodologies, as suggested below:

- Reasonable investment for initial procurement

- Looking for cheaper sources of medical supplies

- Selecting the optimal training model in the context of actual resources

- Learning from international colleagues, especially from those countries with similar resource conditions

Currently, in the age of Information Technology, we are living in an open world. There are numerous opportunities by which we can contact our colleagues all over the world via the internet network or directly through international specialized video conferences for sharing together our experience of different aspects of microsurgery practice, such as clinical experiences, innovations, technical improvements, training models, etc. Educational forums and groups about microsurgery or plastic reconstructive surgery on social networks, like "International Micro-surgery Club" as well as open access journals, like "International Micro-surgery Journal", are offering invaluable opportunities for microsurgical surgeons in developing countries to overcome some of their problems.

\footnotetext{
*Correspondence: Tuan Van Huynh, MD

Department of Burn and Reconstructive Surgery, Trauma and Orthopaedics

Hospital 175, Ho Chi Minh City, Vietnam. Email: bstuan@gmail.com
}

Received: April 18, 2017; Accepted: May 8, 2017; Published: May 9, 2017
International Microsurgery Journal. 2017;1(1):2

DOI: $10.24983 /$ scitemed.imj.2017.00007

Copyright $(2017$ The Author(s). This is an open-access article distributed under the terms of the Creative Commons Attribution 4.0 International License (CC-BY). 